\title{
The influence of heavy physical effort on proteolytic adaptations in skeletal and heart muscle and aorta in rats
}

\author{
Anna Gilbert-Matuszak ${ }^{1, A-F}$, Aleksandra Wyczalkowska-Tomasik ${ }^{2, A-F}$, \\ Malgorzata Zendzian-Piotrowska ${ }^{3, A-F}$, Bozena Czarkowska-Paczek ${ }^{1, A-B, D-F}$ \\ ${ }^{1}$ Department of Clinical Nursing, Medical University, Warsaw, Poland \\ ${ }^{2}$ Department of Immunology, Transplantology and Internal Diseases, Medical University, Warsaw, Poland \\ ${ }^{3}$ Department of Hygiene, Epidemiology and Ergonomics, Medical University, Bialystok, Poland \\ $A$ - research concept and design; $B$ - collection and/or assembly of data; $C$ - data analysis and interpretation; \\ $D$ - writing the article; $E$ - critical revision of the article; $F$ - final approval of article
}

\begin{abstract}
Gilbert-Matuszak A, Wyczalkowska-Tomasik A, Zendzian-Piotrowska M, Czarkowska-Paczek B. The influence of heavy physical effort on
\end{abstract} proteolytic adaptations in skeletal and heart muscle and aorta in rats. Ann Agric Environ Med. 2018; 25(4): 605-609. doi: 10.26444/aaem/74315

\begin{abstract}
Physical effort can elicit differential adaptive changes in the tissues of trained versus untrained rats. Proteolytic activity in the extracellular matrix could be engaged in such adaptation due to its influence on the elasticity of tissues. The effects were investigated of a single physical effort on the activity of elastase, cathepsin $\mathrm{K}$, and plasmin in the skeletal muscles, heart muscles, and aortas of untrained (UT, $n=30)$ and trained $(T, n=30)$ rats. T rats underwent 6 weeks of endurance training. After the last training session, T and UT rats were divided randomly into 3 subgroups. Ten rats from each group (Tpre, $n=10)$ and (UTpre, $n=10$ ) were sacrificed. The other 20 rats from each group performed $60 \mathrm{~min}$. of aerobic exercise and were sacrificed immediately post exercise (T0h, $n=10$; UTOh, $n=10$ ) or 3h later (T3h, $n=10$; UT3h, $n=10$ ). Enzyme activity was measured fluorometrically. Cathepsin K and plasmin activity increased in the soleus muscles of UTOh versus UTpre, plasmin activity increased also in UT3h versus UTpre. Elastase, cathepsin $\mathrm{K}$ and plasmin activity increased in the heart muscles of TOh and T3h versus Tpre. No aortic differences were observed. Thus, a single bout of physical effort elicited different responses in tissues of T versus UT rats. Increased proteolytic enzyme levels in muscles could influence tissue remodeling. Unchanged aortic cathepsin $\mathrm{K}$ levels may help prevent aortic remodeling and neointima formation.
\end{abstract}

\section{Key words}

physical effort, proteolytic enzymes, tissue remodeling

\section{INTRODUCTION}

A single bout of physical effort induces changes in specific tissues and in the whole organism, including increases in oxidative stress, cytokine levels and blood pressure. It may also influence extracellular matrix (ECM) remodeling. The ECM is present in all tissues, providing scaffolding for the surrounding cells and regulating cell behaviour [1]. It plays a role in tissue homeostasis, morphogenesis, and differentiation. The ECM is subjected to rapid remodeling, and there is a delicate balance between the factors that modulate its synthesis and degradation. ECM turnover is involved in cell migration, the formation of myotubes, and tissue regeneration $[2,3]$. There are many enzymes involved in ECM proteolysis. Metalloproteases and serine proteinases are localized extracellularly and are stable at neutral $\mathrm{pH}$, while cysteine cathepsins are lysosomal enzymes that are active at acidic $\mathrm{pH}[3,4]$. ECM remodeling is needed in the processes associated with adaptation to physical effort, such as angiogenesis in skeletal muscles [1]. Notably, the absence of proteolytic enzymes results in abnormal muscle regeneration [5].

Address for correspondence: Bożena Czarkowska-Pączek, Department of Clinical Nursing, Medical University of Warsaw, Erazma Ciolka Street 27, 01-445 Warsaw, Poland

e-mail: bozena.czarkowska-paczek@wum.edu.pl

Received: 17 April 2017; accepted: 1 June 2017; first published on July, 102017
A previous study by the authors showed that 6 -weeks exercise training affects selected proteolytic enzyme activities in skeletal and heart muscles and in the aorta [6]. The aim of this study was to investigate the influence of a single bout of physical effort on the activities of proteolytic enzymes, elastase, cathepsin $\mathrm{K}$, and plasmin in trained and untrained healthy rats in the same tissues studied previously.

\section{MATERIALS AND METHOD}

All procedures used in this study were approved by the Ethics Committee of the Medical University in Bialystok, Poland (Resolution No. 23/2011 on proposal No. 27.04.2011), and were performed in accordance with European Union regulations regarding the humane treatment of laboratory animals.

Rats and exercise protocol. The physical effort protocol was as described in the previous study $[6,7,8]$. A total of 60 male Wistar rats were used. The rats had ad libitum access to water and were fed with Labofeed B under a 12-h light/12-h dark cycle. For the first 5 days, all 60 rats were subjected to exercise adaptation via a once-daily regimen of 10 min of running on a treadmill at $900 \mathrm{~m} / \mathrm{h}$. The rats were then randomly assigned to one of two groups: untrained (UT, $n=30$ ) or trained (T, $n=30$ ). Rats in the trained group were subjected to exercise training 5 days/week for 6 weeks. The exercise intensity and 
duration were gradually increased over time. Initially, the sessions were $10 \mathrm{~min}$ at a pace of $1,200 \mathrm{~m} / \mathrm{h}$; the duration was increased 10 min each day during the first week for a final duration of $60 \mathrm{~min} /$ day, which was maintained over the rest of the training period. The running speed was $1,500 \mathrm{~m} / \mathrm{h}$ in the second week and $1,680 \mathrm{~m} / \mathrm{h}$ for weeks $3-6$. There was no additional running stimulation. The untrained group remained sedentary throughout the training period. The age of the rats at the beginning of exercise was 5-6 weeks.

Twenty-four hours after the last training session, the 2 groups of rats were each randomly divided into 3 subgroups. Ten rats from each group (Tpre, $n=10$ and UTpre, $n=10$ ) were sacrificed without performing any more physical effort. The results from these 2 groups were published previously to report the effects of training on some factors [6]. The remaining 20 rats in each group were subjected to $60 \mathrm{~min}$ of treadmill running at $1,680 \mathrm{~m} / \mathrm{h}$ and then sacrificed at 2 time points: immediately after completion of the physical effort (T0h, $n=10$; UT0h, $n=10$ ) or $3 \mathrm{~h}$ later (T3h, $n=10$; UT3h, $n=10$ ). The relative load was higher for UT rats vs. T rats. The rats were sacrificed under anaesthesia (intraperitoneal chloral hydrate, $1 \mathrm{ml} / 100 \mathrm{mg}$ body mass). Samples of the soleus muscle, heart muscle (ventricle), and aorta were collected and stored immediately at $-80^{\circ} \mathrm{C}$. The soleus muscle was chosen because it has a large proportion of type I slow-twitch fibres [9]. The soleus muscle is primarily recruited during running at the speeds used in this study, while fast-twitch muscles generally are not [10]. The activities of elastase, cathepsin K, and plasmin in both muscle types and aorta were evaluated.

Tissue homogenization and total protein quantification. All samples were homogenized in water in a TissueLyser bead mixer (Qiagen) and centrifuged twice at 10,000 rpm for $10 \mathrm{~min}$ at $4{ }^{\circ} \mathrm{C}$. Plasmin activity and elastase activity were assayed directly after centrifugation. Supernatants were stored at $-80^{\circ} \mathrm{C}$ for further analyses of cathepsin $\mathrm{K}$ and total protein content.
The total protein concentration was measured at $562 \mathrm{~nm}$ on a BioTek Power Wave XS spectrophotometer (BioTek Instruments, USA) using the bicinchoninic acid (BCA) Protein Assay Reagent (Pierce, Holland) in accordance with the manufacturer's instructions.

Assays of enzyme activity. Enzyme activity was measured using a spectrofluorimeter (LS-50B, PerkinElmer, USA). Fluorescence measurements were performed with induction at $\lambda=355 \mathrm{~nm}$ and emission at $\lambda=460 \mathrm{~nm}$. The substrate for elastase activity was Z-Arg-Arg-7-amido-4-methylcoumarin, and the substrate for plasmin was Boc-Val-Leu-Lys-7-amido4-methylcoumarin (Bachem, Biochemica GmbH, Germany). A commercial kit (Cathepsin K Activity Fluorometric Assay Kit, BioVision, Inc., USA) was used to measure cathepsin K activity (substrate: Ac-Lys-Arg-amino-4-trifluoromethyl coumarin) with a 400-nm excitation filter and a 505-nm emission filter. The results are presented as the ratio of enzyme fluorescence to total protein concentration.

Statistical analyses. The results are reported as means \pm standard deviation. Differences in enzyme activity levels between groups were assessed with the Kruskal-Wallis non-parametric ANOVA followed by the Mann-Whitney U test. Bonferroni correction was taken into account. P-values $<0.05$ were considered to be statistically significant.

\section{RESULTS}

In the skeletal muscles (soleus) of untrained rats, cathepsin $\mathrm{K}$ activity was increased immediately after physical effort (UTpre vs. UTOh), and plasmin activity was increased both immediately after physical effeort (UTpre vs. UT0h) and 3 hours after (UTpre vs. UT3h) (Fig. 1). These increases in enzyme activity were not observed in trained rats (Fig. 1).

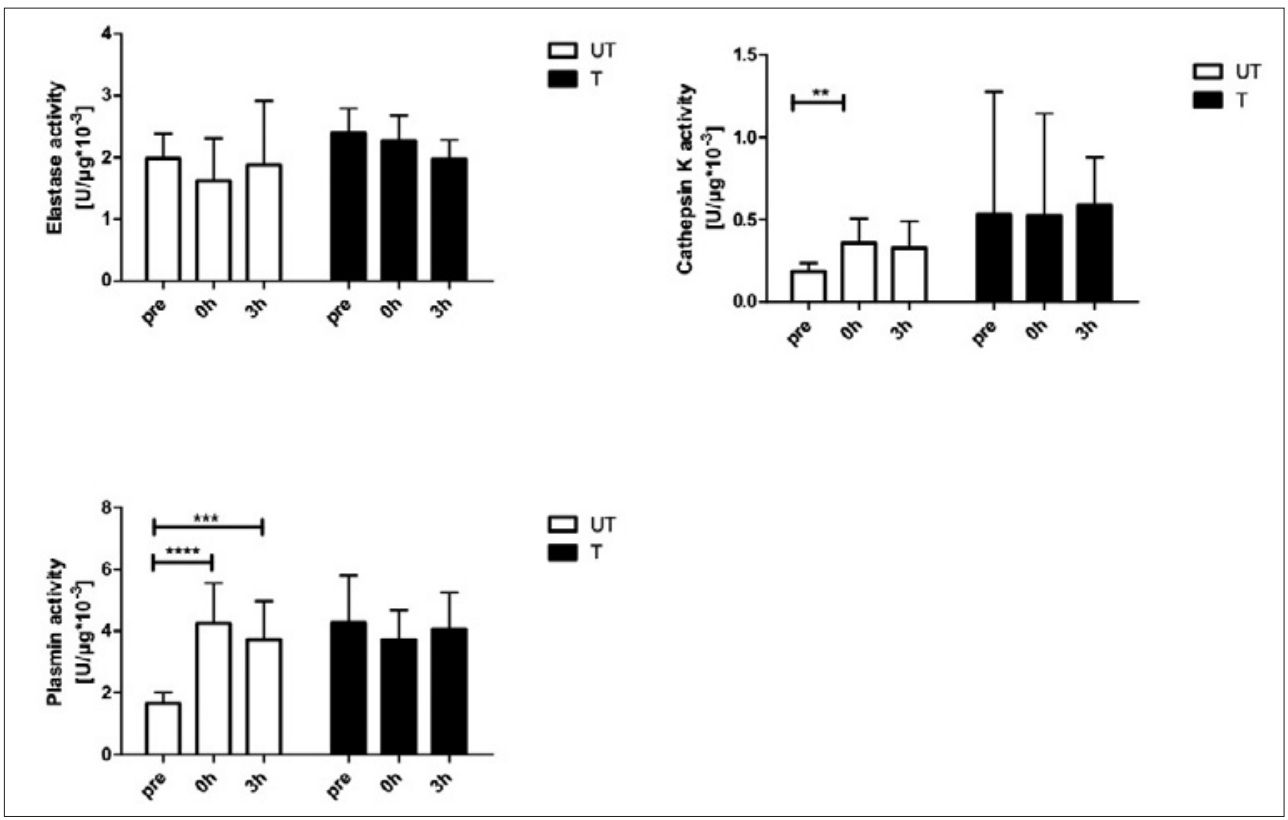

Figure 1. Soleus muscle. The activity of elastase was unchanged in the soleus muscle of untrained rats after a bout of physical effort, while cathepsin Kactivity was increased right after physical effort (UTpre vs. UTOh, $p=0.0036$ ), and plasmin activity was increased both right after physical effort (UTpre vs. UTOh, $p=0.0002$ ) and 3 hours after (UTpre vs. UT3h, $p=0.001008$ ). There were no changes in enzyme activities in this muscle in trained rats after a bout of physical effort. ${ }^{*} p \leq 0.05 ;{ }^{* *} p \leq 0.01 ;{ }^{* * *} p \leq 0.001$; $* * * * \mathrm{p} \leq 0.0001$ 


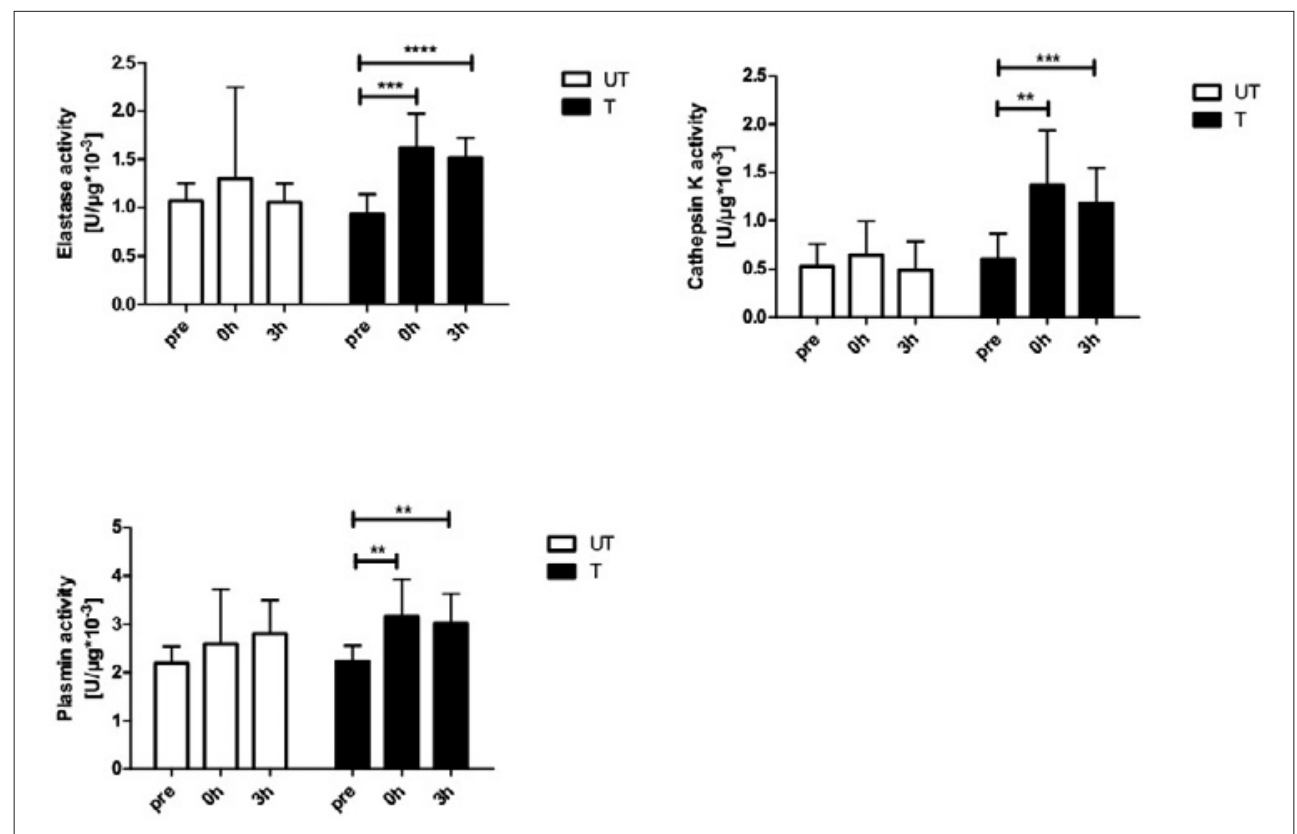

Figure 2. Heart muscle. The activities of elastase, cathepsin $\mathrm{K}$, and plasmin were unchanged in the heart muscle of untrained rats after a bout of physical effort. The activities of elastase, cathepsin $\mathrm{K}$, and plasmin were increased in trained rats right after physical effort (Tpre vs. TOh, $p=0.000769 ; p=0.002497 ; p=0.007285$, respectively) and 3 hours after (Tpre vs. T3h: $p=0.000246$; $p=0.001706 ; p=0.002202$, respectively). ${ }^{*} p \leq 0.05 ;{ }^{* *} p \leq 0.01 ;{ }^{* * *} p \leq 0.001 ; * * * * 0.0001$

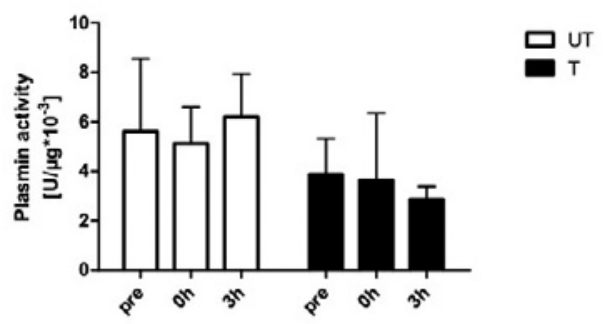

Figure 3. Aorta. The activities of elastase, cathepsin $\mathrm{K}$, and plasmin were unchanged at all time points in all groups of rats

In the heart muscles (ventricles) of untrained rats, proteolytic enzyme activity was not increased immediately after or 3 hours after physical effort. However, there were increases in the activities of proteolytic enzymes (elastase, cathepsin $\mathrm{K}$, and plasmin) in the trained rats, both immediately after physical effort (Tpre vs. T0h) and 3 hours after (Tpre vs. T3h) (Fig. 2).

In the aorta, there were no significant differences in the activity of the proteolytic enzymes (elastase, cathepsin $\mathrm{K}$, and plasmin) either immediately after physical effort or 3 hours after physical effort in either trained or untrained rats (Fig. 3).

\section{DISCUSSION}

The principal finding of this study was that a single bout of physical effort elicited different responses in trained and untrained rats in a tissue-dependent manner. Elastase degrades elastin and other ECM and non-ECM substrates $[4,11]$. Research on elastase content and activity has been conducted mainly in plasma samples. Serteyn et al. found that the elastase levels in muscle did not significantly correlate with the levels in plasma, it is therefore difficult to compare the outcomes of other studies with results of the presented study [12]. Short-duration eccentric exercise, but not concentric exercise, changes the plasma levels of elastase 
in healthy men after 20 min of exercise [13]. In a study by Bishop et al., prolonged intense exercise did not influence the total elastase content of neutrophils in the venous blood of endurance-trained cyclists [14]. Rullman et al. investigated the arterio-venous difference in the concentration of elastase in skeletal muscle and found no difference after 60-min training sessions in healthy men [15]. It appears that both exercise intensity and its' length influence the increase in elastase activity. The exercise protocol in the presented study involved aerobic exercise. In agreement with the study by Rullman et al., no difference was found in elastase activity before physical effort vs. after its cessation in skeletal muscle, but there was an increase in elastase activity in the hearts of trained rats. According to previous research by the authors of the current study, training does not influence the resting activity of elastase in the heart muscle, but it does influence the reaction to a single bout of physical effort. This causes a transient increase in elastase activity, which may be necessary for ECM remodeling [6].

Cathepsin $\mathrm{K}$ is a member of the family of lysosomal cysteine cathepsins and is responsible for the proteolysis activity in ECM. It also plays a role in the immune response, in bone remodeling, and in prohormone processing [3]. Cathepsin $\mathrm{K}$ is one of the most potent elastases, and it also functions as a collagenase. The authors suspect that it may play a role in the prevention of fibrosis [4]. In the current study, cathepsin $\mathrm{K}$ activity increased immediately after physical effort in both muscles, and this increase was maintained 3 hours later in the heart muscle. This increase was observed in the soleus muscle of untrained rats and in the heart muscle of trained rats. Because the increases in cathepsin $\mathrm{K}$ activity were found along with increased elastin level in trained rats [6], it is suspected that cathepsin plays a role in extracellular proteolysis, and thus has adaptive potential.

Plasmin is the main enzyme responsible for fibrinolysis. It degrades a number of ECM proteins, either directly or by activating matrix metalloproteinases. Plasmin also plays a role in the inflammatory response in terms of the release of lipid mediators, in chemotaxis, and in the expression of proinflammatory genes $[16,17]$. Plasmin is formed by the conversion of plasminogen into plasmin by t-PA [18]. In the presented study, plasmin activity increased in response to physical effort in the skeletal muscle of untrained rats, reaching a mean level that was similar to the mean resting level in trained rats. In the heart muscle, plasmin activity only increased in trained rats, but not in untrained rats, after the bout of physical effort. The increase in plasmin activity is clearly beneficial, especially in heart muscle, as it prevents clotting. Plasmin is also involved in myogenesis, muscle regeneration and hypertrophy $[19,20]$.

The aorta needs to be elastic to serve as a buffering vessel. Studies have shown that training prevents aorta stiffening $[21,22]$. There must be different mechanisms that provide aorta elasticity, since this study shows that a bout of physical effort does not influence proteolytic enzyme activity in the aorta. However, the lack of increase in cathepsin K activity could be beneficial. Cathepsin $\mathrm{K}$ expression is increased in human and animal atherosclerotic lesions, and cathepsin $\mathrm{K}$ plays an essential role in neointimal lesion formation in response to injury [23].

The ECM plays crucial roles both in skeletal muscle and in cardiac muscle in force transmission and in structural support. It is also a reservoir of a number of proteins, such as growth factors and cell receptor-binding proteins [24, 25]. Notably, excessive levels of some substances in the ECM can be disruptive as they can lead to fibrosis and then to passive stiffness of the muscle [24]. In the presented study, the postexercise changes in proteolytic enzymes were different in the skeletal muscle vs. the heart muscle. The authors reported previously that the activities of proteolytic enzymes are increased in soleus muscle of trained rats (Tpre) vs. untrained rats (UTpre) [6]. The results reported here show that there are no further increases after a single bout of physical effort in trained rats. On the other hand, proteolytic activity only increases in the heart muscle after a bout of physical effort in rats that have trained for 6 weeks. These observations may indicate that after 6 -week endurance training, a proteolytic activity plateau is achieved in skeletal muscle, but not in the heart ventricle. These changes seem to have adaptive potential, as ECM proteolysis is necessary for the infiltration of fibroblasts, inflammatory cells, and macrophages. It also allows the removal of necrotic tissue and the initiation of revascularization and of satellite cell proliferation [19].

\section{CONCLUSIONS}

The results obtained indicate that physical effort activates different signaling pathways in different tissues, depending on training status. Proteolytic enzymes activity shows different patterns in skeletal vs. heart muscle. Skeletal muscles show increases in cathepsin $\mathrm{K}$ and plasmin activity in untrained rats, and increases in the activity of 3 proteolytic enzymes in the heart muscles of trained rats. As ECM remodeling is needed for muscle regeneration, it can be concluded that these changes reflect adaptive potential.

\section{REFERENCES}

1. Suhr F, Brixius K, Bloch W. Angiogenic and vascular modulation by extracellular matrix cleavage products. Curr Pharm Des. 2009; 15(4): 389-410.

2. Gillies AR, Lieber RL. Structure and Function of the Skeletal Muscle Extracellular Matrix. Muscle Nerve. 2011; 44(3): 318.

3. Fonović M, Turk B. Cysteine cathepsins and extracellular matrix degradation. Biochim Biophys Acta. 2014; 1840(8): 2560-2570.

4. Antonicelli F, Bellon G, Debelle L, Hornebeck W. Elastin-elastases and inflamm-aging. Curr Top Dev Biol. 2007; 79: 99-155.

5. Vinarsky V, Atkinson DL, Stevenson TJ, Keating MT, Odelberg SJ. Normal newt limb regeneration requires matrix metalloproteinase function. Dev Biol. 2005; 279(1): 86-98.

6. Gilbert A, Wyczalkowska-Tomasik A, Zendzian-Piotrowska M, Czarkowska-Paczek B. Training differentially regulates elastin level and proteolysis in skeletal and heart muscles and aorta in healthy rats. Biol Open. 2016: bio.017459.

7. Czarkowska-Paczek B, Zendzian-Piotrowska M, Gala K, Sobol M, Paczek L. One session of exercise or endurance training does not influence serum levels of irisin in rats. J Physiol Pharmacol Off J Pol Physiol Soc. 2014; 65(3): 449-454.

8. Czarkowska-Paczek B, Zendzian-Piotrowska M, Bartlomiejczyk I, Przybylski J, Gorski J. Skeletal and heart muscle expression of PDGF-AA and VEGF-A after an acute bout of exercise and endurance training in rats. Med Sci Monit Int Med J Exp Clin Res. 2010; 16(5): BR147-153.

9. Feng H-Z, Chen M, Weinstein LS, Jin J-P. Improved fatigue resistance in Gsa-deficient and aging mouse skeletal muscles due to adaptive increases in slow fibers. J Appl Physiol Bethesda Md. 1985; 2011; 111(3): 834-843.

10. Lambert MI, Noakes TD. Dissociation of changes in VO2 max, muscle QO2, and performance with training in rats. J Appl Physiol Bethesda Md. 1985, 1989; 66(4): 1620-1625. 
11. Paczek L, Michalska W, Bartlomiejczyk I. Trypsin, elastase, plasmin and MMP-9 activity in the serum during the human ageing process. Age Ageing. 2008; 37(3): 318-323.

12. Serteyn D, Sandersen C, Lejeune J-P, Rebière de Pouyade G de la, Ceusters J, Mouithys-Mickalad A et al. Effect of a $120 \mathrm{~km}$ endurance race on plasma and muscular neutrophil elastase and myeloperoxidase concentrations in horses. Equine Vet J Suppl. 2010; (38): 275-279.

13. Camus G, Pincemail J, Ledent $M$, Juchmès-Ferir A, Lamy M, DebyDupont G, et al. Plasma levels of polymorphonuclear elastase and myeloperoxidase after uphill walking and downhill running at similar energy cost. Int J Sports Med. 1992; 13(6): 443-446.

14. Bishop NC, Walsh NP, Scanlon GA. Effect of prolonged exercise and carbohydrate on total neutrophil elastase content. Med Sci Sports Exerc. 2003; 35(8): 1326-1332.

15. Rullman E, Olsson K, Wågsäter D, Gustafsson T. Circulating MMP-9 during exercise in humans. Eur J Appl Physiol. 2013; 113(5): 1249-1255.

16. Syrovets T, Simmet T. Novel aspects and new roles for the serine protease plasmin. Cell Mol Life Sci CMLS. 2004; 61(7-8): 873-885.

17. Li Q, Laumonnier Y, Syrovets T, Simmet T. Plasmin triggers cytokine induction in human monocyte-derived macrophages. Arterioscler Thromb Vasc Biol. 2007; 27(6): 1383-1389.

18. Kupchak BR, Volk BM, Kunces LJ, Kraemer WJ, Hoffman MD, Phinney $\mathrm{SD}$, et al. Alterations in coagulatory and fibrinolytic systems following an ultra-marathon. Eur J Appl Physiol. 2013; 113(11): 2705-2712.
19. Suelves M, López-Alemany R, Lluís F, Aniorte G, Serrano E, Parra M et al. Plasmin activity is required for myogenesis in vitro and skeletal muscle regeneration in vivo. Blood. 2002; 99(8): 2835-2844.

20. López-Alemany R, Suelves M, Muñoz-Cánoves P. Plasmin generation dependent on alpha-enolase-type plasminogen receptor is required for myogenesis. Thromb Haemost. 2003; 90(4): 724-733.

21. Shibata S, Levine BD. Effect of exercise training on biologic vascular age in healthy seniors. Am J Physiol Heart Circ Physiol. 2012; 302(6): H1340-1346.

22. Fleenor BS, Marshall KD, Durrant JR, Lesniewski LA, Seals DR. Arterial stiffening with ageing is associated with transforming growth factor$\beta 1$-related changes in adventitial collagen: reversal by aerobic exercise. J Physiol. 2010; 588(Pt 20): 3971-3982.

23. Hu L, Cheng XW, Song H, Inoue A, Jiang H, Li X et al. Cathepsin K activity controls injury-related vascular repair in mice. Hypertension. 2014; 63(3): 607-615.

24. Spinale FG, Janicki JS, Zile MR. Membrane Associated Matrix Proteolysis and Heart Failure. Circ Res. 2013; 112(1): 195.

25. Rienks M, Papageorgiou A-P, Frangogiannis NG, Heymans S. Myocardial Extracellular Matrix An Ever-Changing and Diverse Entity. Circ Res. 2014; 114(5): 872-888. 